Article

\title{
Synthesis and Herbicidal Activity of 5-Heterocycloxy-3-methyl-1-substituted-1H-pyrazoles
}

\author{
Jing Kang ${ }^{1}$, Xia Li Yue ${ }^{1}$, Chang Shui Chen ${ }^{1}$, Jian Hong $\mathrm{Li}^{2}$ and Hong Ju Ma ${ }^{2, *}$ \\ Received: 17 August 2015 ; Accepted: 11 December 2015 ; Published: 25 December 2015 \\ Academic Editor: Jean Jacques Vanden Eynde \\ 1 Department of Applied Chemistry, College of Science, Huazhong Agricultural University, Wuhan 430070, \\ China; kangjing@webmail.hzau.edu.cn (J.K.); yxl@mail.hzau.edu.cn (X.L.Y.); \\ chenchang@mail.hzau.edu.cn (C.S.C.) \\ 2 Department of Plant Protection, College of Plant Science and Technology, \\ Huazhong Agricultural University, Wuhan 430070, China; jianhl@mail.hzau.edu.cn \\ * Correspondence: mahongju@mail.hzau.edu.cn; Tel.: +86-278-728-6968
}

\begin{abstract}
With the objective of finding valuable herbicidal candidates, a series of new 5-heterocycloxy3-methyl-1-substituted-1H-pyrazoles were synthesized and their herbicidal activities were evaluated. The bioassay results showed that some compounds exhibited excellent herbicidal activities at the concentration of $100 \mathrm{mg} / \mathrm{L}$, and compound 5-chloro-2-((3-methyl-1-(2,2,2-trifluoroethyl)-1H-pyrazol5-yl)oxy)pyrimidine showed bleaching activity to green weeds. In greenhouse conditions, this compound also showed excellent post-emergence herbicidal effect against Digitaria sanguinalis L. at the dosage of $750 \mathrm{~g}$ a. i. ha ${ }^{-1}$.
\end{abstract}

Keywords: pyrazole; synthesis; herbicidal activities

\section{Introduction}

Pyrazole derivatives not only occupy an important position in medicinal chemistry due to their wide range of bioactivities such as anticancer [1], analgesic [2], anti-convulsant [3], anti-depressant [4], anti-inflammatory [5], antibacterial [6] antimalarial [6], and antituberculosis activity [6], but also has been drawn great attention in agrochemicals because of their excellent bioactivity such as the commercialized herbicides pyrazolate, pyrazoxyfen, benzofenap, pyraflufen-ethyl, fluazolate, and pyrazosulfuron-ethyl [7-15]. Owing to the interesting applications of pyrazoles in the field of agricultural research, the combination of such pyrazole molecule with the additional heterocycles to form polycyclic systems to add functional diversity, is increasingly becoming a fruitful area of the study for their biological activity [16]. Compounds with fused heterocycles showed excellent bioactivity, such as metamifop, fenoxaprop, and pyriftalid $[17,18]$. Plants treated with herbicides inhibiting carotenoid biosynthesis show characteristic white foliage. Carotenoids protect chlorophyll from photooxidation and chlorophyll is destroyed as it is formed in tissues being devoid of carotenoids. One of the well-studied sites of carotenoid biosynthesis inhibition is that of phytoene desaturase (PDS) and many reviews have been published [19-26]. Despite the great number of structurally diverse inhibitors of phytoene desaturase have been known, the enzyme is still a good target site for new herbicides owing to their good selective toxicity. This has led to the discovery of 2-((5-methyl-3'-(trifluoromethyl)-[1,1'-biphenyl]-2-yl)oxy)-5-(trifluoromethyl)-1,3,4-thiadiazole (1) or 6-(benzothiazol-2-yloxy)-3'-(trifluoromethyl)-[1,1'-biphenyl]-3-carbaldehyde (2) and 5-chloro-2phenyl-7-(3-(trifluoromethyl)phenyl)benzoxazole (3) as bleaching herbicides candidates [23,27]. The introduction of trifluoromethyl into $N$-methyl group of pyrazole ring would be expected to improve herbicidal activity due to the intrinsic properties of trifluoromethyl, such as high thermal stability, increased lipophilicity, its electronegativity, and relatively small size $[28,29]$. The chemical structures 
of the compounds mentioned above was represented in Figure 1. The pyrazolylpyrimidine derivatives have been reported to show inhibition of chlorophyll and carotenoid biosynthesis in our previous work [30,31]. In view of the above mentioned facts and in continuation of our interest in the synthesis of pyrazole heterocycles, the synthesis and herbicidal activities of these novel pyrazole derivatives are described in this paper.

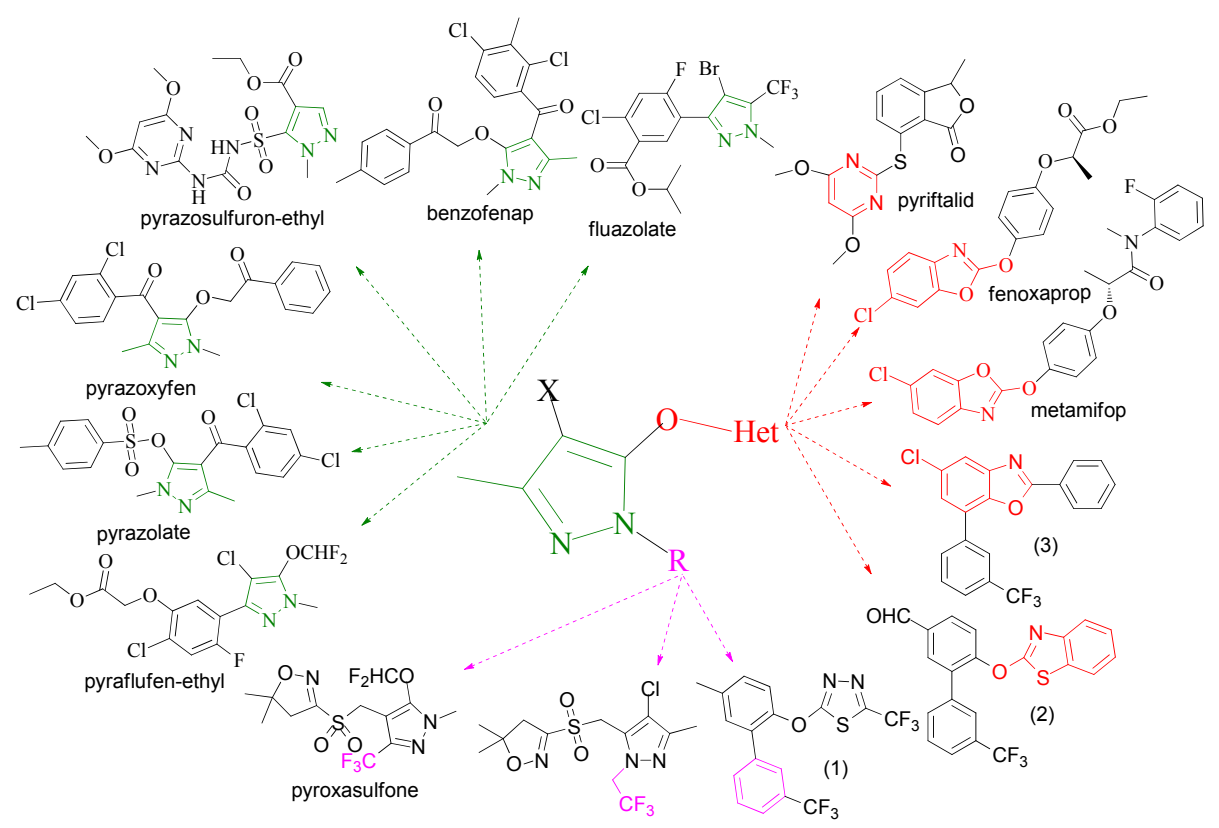

Figure 1. Structural importance of few of the molecules.

\section{Results and Discussion}

\subsection{Synthesis}

The synthetic route of a series of pyrazole derivatives were shown in Scheme 1 . The chemical structures of fourteen target compounds were listed in Table 1 . The structures of these compounds were confirmed by ${ }^{1} \mathrm{H}-\mathrm{NMR}$ (see Supplementary Materials), ${ }^{13} \mathrm{C}-\mathrm{NMR}$ (see Supplementary Materials), MS, Elemental analysis, and FT-IR. The compound $\mathbf{5 b}$ was synthesized according to the method of preparing 3,5-dimethyl-1-(2,2,2-trifluoroethyl)-1-H-pyrazole reported in the literature [29].

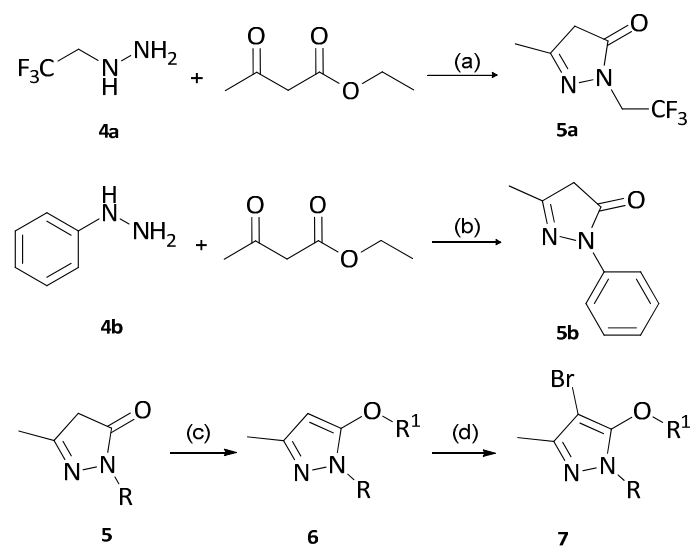

Scheme 1. Synthetic route of target compounds. Reagents and conditions: (a) $\mathrm{C}_{2} \mathrm{H}_{5} \mathrm{OH}$, reflux, $3 \mathrm{~h}$; (b) $\mathrm{C}_{2} \mathrm{H}_{5} \mathrm{OH} / \mathrm{H}_{2} \mathrm{O}=1: 2, \mathrm{HCl}, \mathrm{rt}, 5 \mathrm{~min}, 60^{\circ} \mathrm{C}, 1.5 \mathrm{~h}, \mathrm{pH}=7$; (c) $\mathrm{R}^{1}-\mathrm{Cl}$, DMSO, $\mathrm{K}_{2} \mathrm{CO}_{3}, 3$ h; (d) NBS, $\mathrm{DMF}, \mathrm{rt}$, overnight. 
Table 1. Chemical structure of target compounds

Compd.

\subsection{Growth Inhibition of Weed Roots and Shoots}

The herbicidal activities of the target compounds were determined with Brassica campestris $\mathrm{L}$. (B. campestris), Amaranthus retroflexus L. (A. retroflexus) and Portulaca oleracea L. (P. oleracea) as samples of annual dicotyledonous plants and Pennisetum alopecuroides L. (P. alopecuroides), Echinochloa crus-galli L. (E. crus-galli), and Digitaria sanguinalis L. (D. sanguinalis) as samples of annual monocotyledonous plants. The results of the inhibition effect were shown in Table 2. All compounds showed inhibitory effect on the roots growth of B. campestris, A. retroflexus, P. oleracea, and D. sanguinalis and the shoots growth of $P$. alopecuroides and E. crus-galli. Compounds $\mathbf{6 d}$ and $\mathbf{7 d}$ showed excellent inhibition effect against all tested dicotyledonous and monocotyledonous plants at $100 \mathrm{mg} / \mathrm{L}$. Compound $\mathbf{6 d}$ exhibited excellent bleaching activities to B. campestris, P. alopecuroides, E. crus-galli, and D. sanguinalis, and even bleached $D$. sanguinalis at the concentration of $10 \mathrm{mg} / \mathrm{L}$. Among these target compounds, only compound $\mathbf{6 d}$ showed best bleaching activity. It was possible that pyrimidine group played a key role in bleaching phytotoxicity. Comparing the compounds $\mathbf{6 d}$ and $\mathbf{7 d}$, the presence of bromine at the 4-position on the pyrazole ring had a negative effect on bleaching activity.

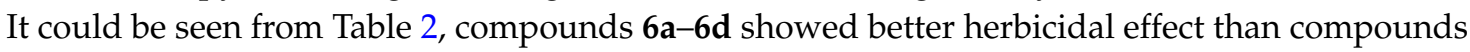
6e-6i. The derivatives with 2,2,2-trifluoroethyl at the 1-position on pyrazole showed better inhibition effect than that of compounds substituted with the phenyl group, indicating the 2,2,2-trifluoroethyl group on the pyrazole ring played an important role in inhibition. Compounds $\mathbf{6} \mathbf{d}$ and $\mathbf{6 h}$ had better inhibition activities than compounds $6 \mathbf{a}-6 \mathbf{c}$ and $6 \mathrm{e}-6 \mathrm{~g}$, indicating that the bulkiness of fused heterocyclic substitute at pyrazole ring might be attributable to the decreased of herbicidal activity. Comparing the activities of compounds 6 and compounds 7 , the electron-withdrawing bromine group at C-4 had no beneficial effect on activity. 
Table 2. Inhibition of target compounds on the growth of weed.

\begin{tabular}{|c|c|c|c|c|c|c|c|c|c|c|c|c|}
\hline \multirow{4}{*}{ Compd. } & \multicolumn{12}{|c|}{ Relative Inhibition (\%) } \\
\hline & \multirow{2}{*}{\multicolumn{2}{|c|}{$\begin{array}{c}\text { B. campestris } \\
\text { Root }\end{array}$}} & \multirow{2}{*}{\multicolumn{2}{|c|}{$\begin{array}{c}\text { A. retroflexus } \\
\text { Root }\end{array}$}} & \multirow{2}{*}{\multicolumn{2}{|c|}{$\begin{array}{l}\text { P. oleracea } \\
\text { Root }\end{array}$}} & \multirow{2}{*}{\multicolumn{2}{|c|}{$\begin{array}{c}\text { P. alopecuroides } \\
\text { Shoot }\end{array}$}} & \multirow{2}{*}{\multicolumn{2}{|c|}{$\begin{array}{c}\text { E. crus-galli } \\
\text { Shoot }\end{array}$}} & \multirow{2}{*}{\multicolumn{2}{|c|}{$\begin{array}{c}\text { D. sanguinalis } \\
\text { Root }\end{array}$}} \\
\hline & & & & & & & & & & & & \\
\hline & $10 \mathrm{mg} / \mathrm{L}$ & $100 \mathrm{mg} / \mathrm{L}$ & $10 \mathrm{mg} / \mathrm{L}$ & $100 \mathrm{mg} / \mathrm{L}$ & $10 \mathrm{mg} / \mathrm{L}$ & $100 \mathrm{mg} / \mathrm{L}$ & $10 \mathrm{mg} / \mathrm{L}$ & $100 \mathrm{mg} / \mathrm{L}$ & $10 \mathrm{mg} / \mathrm{L}$ & $100 \mathrm{mg} / \mathrm{L}$ & $10 \mathrm{mg} / \mathrm{L}$ & $100 \mathrm{mg} / \mathrm{L}$ \\
\hline $6 a$ & $69 \pm 2.1$ & $78 \pm 1.1$ & $39 \pm 1.1$ & $58 \pm 2.1$ & $3 \pm 1.3$ & $48 \pm 1.6$ & $47 \pm 1.0$ & $59 \pm 0.5$ & $47 \pm 1.1$ & $57 \pm 2.3$ & $27 \pm 1.5$ & $58 \pm 1.1$ \\
\hline $6 b$ & $80 \pm 1.2$ & $81 \pm 1.1$ & $53 \pm 1.7$ & $60 \pm 2.4$ & $29 \pm 1.2$ & $50 \pm 0.8$ & $56 \pm 2.2$ & $59 \pm 1.6$ & $60 \pm 0.6$ & $70 \pm 1.3$ & $31 \pm 2.9$ & $75 \pm 0.7$ \\
\hline $6 c$ & $37 \pm 1.5$ & $46 \pm 2.0$ & $26 \pm 3.3$ & $58 \pm 1.5$ & $30 \pm 1.7$ & $74 \pm 2.1$ & $38 \pm 2.7$ & $55 \pm 1.0$ & $26 \pm 1.4$ & $61 \pm 0.6$ & $9 \pm 2.4$ & $21 \pm 0.8$ \\
\hline $6 d$ & $49 \pm 2.4$ & $\bar{W}$ & $41 \pm 0.3$ & 100 & $42 \pm 1.8$ & 100 & $34 \pm 1.6$ & $\bar{W}$ & $30 \pm 2.0$ & $\overline{\mathrm{W}}$ & W & $\bar{W}$ \\
\hline $6 e$ & $21 \pm 0.5$ & $22 \pm 1.6$ & $0 \pm 1.2$ & $27 \pm 2.5$ & $4 \pm 2.2$ & $32 \pm 1.9$ & $22 \pm 1.2$ & $32 \pm 1.2$ & $33 \pm 2.4$ & $36 \pm 2.7$ & $2 \pm 1.4$ & $28 \pm 0.7$ \\
\hline $6 f$ & $42 \pm 1.3$ & $54 \pm 2.1$ & $0 \pm 1.3$ & $7 \pm 1.7$ & $3 \pm 2.0$ & $23 \pm 2.0$ & $30 \pm 1.9$ & $58 \pm 1.9$ & $30 \pm 2.4$ & $52 \pm 0.5$ & $36 \pm 1.3$ & $46 \pm 1.3$ \\
\hline $6 g$ & $16 \pm 1.0$ & $36 \pm 1.0$ & $3 \pm 1.8$ & $13 \pm 0.3$ & $11 \pm 0.5$ & $53 \pm 0.7$ & $21 \pm 1.4$ & $34 \pm 1.1$ & $12 \pm 1.6$ & $24 \pm 1.5$ & $2 \pm 4.4$ & $7 \pm 3.5$ \\
\hline $6 h$ & $17 \pm 1.6$ & $62 \pm 3.0$ & $9 \pm 0.2$ & $35 \pm 1.5$ & $41 \pm 1.0$ & $66 \pm 0.7$ & $40 \pm 1.9$ & $63 \pm 1.6$ & $29 \pm 1.5$ & $53 \pm 0.8$ & $1 \pm 1.5$ & W \\
\hline $6 i$ & $61 \pm 1.4$ & $64 \pm 0.5$ & $6 \pm 1.1$ & $29 \pm 1.2$ & $3 \pm 2.6$ & $32 \pm 0.9$ & $48 \pm 1.3$ & $54 \pm 1.7$ & $31 \pm 1.4$ & $37 \pm 2.1$ & $9 \pm 2.8$ & $22 \pm 2.2$ \\
\hline $7 a$ & $27 \pm 1.4$ & $59 \pm 2.4$ & $25 \pm 2.2$ & $53 \pm 0.6$ & $11 \pm 2.2$ & $55 \pm 0.5$ & $24 \pm 2.8$ & $41 \pm 0.8$ & $27 \pm 2.3$ & $54 \pm 2.3$ & $32 \pm 2.4$ & $74 \pm 0.3$ \\
\hline $7 b$ & $36 \pm 1.7$ & $53 \pm 0.9$ & $39 \pm 1.0$ & $58 \pm 1.0$ & $34 \pm 0.7$ & $61 \pm 0.4$ & $48 \pm 1.7$ & $78 \pm 1.7$ & $53 \pm 1.7$ & $78 \pm 1.7$ & $44 \pm 1.9$ & $67 \pm 1.9$ \\
\hline $7 c$ & $22 \pm 1.2$ & $72 \pm 0.5$ & $8 \pm 3.5$ & $20 \pm 2.1$ & $25 \pm 1.8$ & $49 \pm 2.0$ & $37 \pm 2.1$ & $56 \pm 1.4$ & $18 \pm 0.1$ & $68 \pm 0.2$ & $28 \pm 2.1$ & $63 \pm 0.2$ \\
\hline $7 d$ & $46 \pm 1.6$ & $79 \pm 0.5$ & $46 \pm 1.8$ & $79 \pm 1.0$ & $48 \pm 2.1$ & $72 \pm 0.8$ & $49 \pm 2.2$ & $77 \pm 0.6$ & $22 \pm 0.6$ & $83 \pm 1.1$ & $45 \pm 1.5$ & $77 \pm 0.7$ \\
\hline $7 e$ & $32 \pm 3.5$ & $64 \pm 1.7$ & $4 \pm 0.7$ & $15 \pm 0.7$ & $3 \pm 1.3$ & $23 \pm 1.4$ & $32 \pm 1.5$ & $61 \pm 0.4$ & $21 \pm 1.7$ & $43 \pm 2.5$ & $13 \pm 1.4$ & $22 \pm 2.5$ \\
\hline
\end{tabular}

W: Leaves were completely white after treatment; B. campestris, Brassica campestris L.; A. retroflexus, Amaranthus retroflexus L.; P. oleracea, Portulaca oleracea L.; P. alopecuroides, Pennisetum alopecuroides L.; E. crus-galli, Echinochloa crus-galli L.; D. sanguinalis, Digitaria sanguinalis L. 


\subsection{Screening in Greenhouse Conditions}

Seven target compounds $\mathbf{6 a}-\mathbf{6 d}, \mathbf{7 a}, \mathbf{7 b}, \mathbf{7 d}$ with higher inhibitory effects on the growth of tested plants in preliminary herbicidal bioassays were further screened in greenhouse conditions. From the biological assay results in Table 3, each compound showed herbicidal activities in postemergence treatment at the dosage of $750 \mathrm{~g}$ a. i. ha ${ }^{-1}$, especially monocotyledonous weed $D$. sanguinalis was most sensitive to compound $\mathbf{6 d}$. It was also found that when the 5-position of the pyrazole was modified by a pyrimidine group, compound $\mathbf{6} \mathbf{d}$ had better inhibitory effect on $D$. sanguinalis than other target compounds, the inhibition rate of the fresh weights reaching $82 \%$. The bioassay results indicated that the substituted group at the 5-position of the pyrazole ring played an important role for herbicidal activity. The 4-[5-methyl-3-(trifluoromethyl)-1H-pyrazol-1-yl]-6-(prop-2-yn-1-yloxy)pyrimidine reported in our previous work [30] was very close to compound $\mathbf{6 d}$, structurally, but it showed less herbicidal activity than compound $\mathbf{6} \mathbf{d}$ in greenhouse conditions, the difference between these two structures lay in that the pyrimidine was substituted at the 1-position of the pyrazole ring [30] and at the 5-position of the pyrazole ring in this paper, we might conclude that the substituted position on the pyrazole ring also played an important role for herbicidal activity.

Table 3. Herbicidal activities of compounds in greenhouse conditions

\begin{tabular}{ccccccc}
\hline \multirow{2}{*}{ Compd. } & \multicolumn{6}{c}{ Relative Inhibition (\%) } \\
\cline { 2 - 6 } & $\begin{array}{c}\boldsymbol{A} \text {. } \\
\text { theophrasti }\end{array}$ & $\begin{array}{c}\boldsymbol{A} \text {. } \\
\text { retroflexus }\end{array}$ & $\begin{array}{c}\boldsymbol{P} \text { oleracea } \\
\text { olopecuroides }\end{array}$ & $\begin{array}{c}\boldsymbol{E} \text {. } \\
\text { crus-galli }\end{array}$ & $\begin{array}{c}\text { D. } \\
\text { sanguinalis }\end{array}$ \\
\hline 6a & $22 \pm 1.3$ & $27 \pm 1.4$ & $30 \pm 1.2$ & $42 \pm 1.4$ & $34 \pm 2.6$ & $21 \pm 2.7$ \\
6b & $35 \pm 2.0$ & $29 \pm 0.7$ & $40 \pm 1.1$ & $25 \pm 0.8$ & $26 \pm 2.2$ & $17 \pm 2.0$ \\
6c & $40 \pm 1.0$ & $44 \pm 0.3$ & $38 \pm 1.7$ & $32 \pm 1.6$ & $30 \pm 1.6$ & $33 \pm 2.6$ \\
6d & $42 \pm 2.0$ & $50 \pm 0.9$ & $30 \pm 0.7$ & $6 \pm 2.7$ & $41 \pm 2.6$ & $82 \pm 0.9$ \\
7a & $21 \pm 0.6$ & $46 \pm 1.6$ & $34 \pm 2.6$ & $12 \pm 3.1$ & $60 \pm 0.5$ & $56 \pm 0.6$ \\
7b & $9 \pm 0.6$ & $56 \pm 0.9$ & $34 \pm 1.5$ & $5 \pm 2.1$ & $50 \pm 2.3$ & $46 \pm 1.7$ \\
7d & $11 \pm 2.2$ & $37 \pm 1.8$ & $28 \pm 1.3$ & $17 \pm 2.7$ & $48 \pm 0.4$ & $52 \pm 0.3$ \\
\hline
\end{tabular}

A. theophrasti, Abutilon theophrasti M.; A. retroflexus, Amaranthus retroflexus L.; P. oleracea, Portulaca oleracea L.; P. alopecuroides, Pennisetum alopecuroides L.; E. crus-galli, Echinochloa crus-galli L.; D. sanguinalis, Digitaria sanguinalis L.

\subsection{Inhibitory Effect of the Compound $\mathbf{6 d}$ on Chlorophyll of Weed}

In order to evaluate the bleaching activities of compound $\mathbf{6} \mathbf{d}$, the changes in chlorophyll contents of treated seedlings were tested. The $\mathrm{IC}_{50}$ value of compound $\mathbf{6 d}$ and positive control (diflufenican) were shown in Table 4. Compound $\mathbf{6 d}$ inhibited the synthesis of chlorophyll, and showed the same inhibition activity as commercial standard diflufenican against B. campestris. Gramineous weed P. alopecuroides, E. crus-galli, and D. sanguinalis were slightly sensitive to diflufenican than compound $\mathbf{6 d}$ and compound $\mathbf{6 d}$ deserved further studies on structure optimization and biological efficacy as the bleaching herbicidal inhibitor.

Table 4. Determination of chlorophyll inhibition of compound $\mathbf{6 d}$.

\begin{tabular}{|c|c|c|c|c|}
\hline \multirow[b]{2}{*}{ Species } & \multicolumn{2}{|l|}{$6 d$} & \multicolumn{2}{|c|}{ Diflufenican } \\
\hline & $\begin{array}{l}\text { IC }_{50}(95 \% \text { Confidence } \\
\text { Intervals })\left(\mathrm{mg} \cdot \mathrm{L}^{-1}\right)\end{array}$ & Slope $( \pm$ SE) & $\begin{array}{l}\text { IC }_{50}(95 \% \text { Confidence } \\
\text { Intervals) }\left(\mathrm{mg} \cdot \mathrm{L}^{-1}\right)\end{array}$ & Slope $( \pm$ SE $)$ \\
\hline B. campestris & $20.01(16.48-24.28)$ & $3.54 \pm 0.32$ & $19.79(11.23-34.90)$ & $1.15 \pm 0.39$ \\
\hline P. alopecuroides & $11.71(10.57-12.98)$ & $1.53 \pm 0.08$ & $5.97(4.04-8.82)$ & $0.45 \pm 0.06$ \\
\hline E. crus-galli & $6.14(5.83-6.46)$ & $2.09 \pm 0.04$ & $0.88(0.07-9.81)$ & $2.24 \pm 0.28$ \\
\hline D. sanguinalis & $8.09(6.08-10.76)$ & $4.17 \pm 0.34$ & $1.09(0.36-3.24)$ & $3.12 \pm 0.16$ \\
\hline
\end{tabular}

B. campestris, Brassica campestris L.; P. alopecuroides, Pennisetum alopecuroides L.; E. crus-galli, Echinochloa crus-galli L.; D. sanguinalis, Digitaria sanguinalis L. 


\section{Experimental Section}

\subsection{Analysis and Instruments}

Elemental analyses $(\mathrm{C}, \mathrm{H}, \mathrm{N})$ were performed with a Vario EL III elemental analyzer (Elementar Analysensysteme Gmbh, Hanau, Germany) at the Institute of Chemistry, Chinese Academy of Sciences. Infrared spectra were taken on a Nicolet IR 200 FT-IR instrument (Thermo Scientific, Waltham, MA, USA). ${ }^{1} \mathrm{H}-\mathrm{NMR}$ spectra and ${ }^{13} \mathrm{C}-\mathrm{NMR}$ were obtained at $400 \mathrm{MHz}$ using a Bruker AVANCE III 400 spectrometer (Bruker Corp., Billerica, MA, USA) in $\mathrm{CDCl}_{3}$ solution with TMS as an internal standard. Mass spectra (MS) were recorded in a Agilent 6110 spectrometer (Agilent, Santa Clara, CA, USA). Melting points were measured on a SGW X-4 melting point apparatus (Shanghai Precision Scientific Instrument Co., Ltd., Shanghai, China) and are uncorrected. All reagents and solvents were obtained from commercial suppliers.

\subsection{Synthesis and Characterization of Target Compounds}

\subsubsection{Synthesis of Compounds $\mathbf{5 a}$ and $\mathbf{5 b}$}

A solution of 2,2,2-trifluoroethylhydrazine ( $0.03 \mathrm{~mol}, 70 \%$ in water) and ethyl acetoacetate $(0.03 \mathrm{~mol})$ in ethanol $(50 \mathrm{~mL})$ was stirred at $60{ }^{\circ} \mathrm{C}$ for $3 \mathrm{~h}$. The solvent was removed in vacuo, and the resulting residue was purified by silica gel column chromatography (ethyl acetate/petroleum ether: $1 / 4$ as the eluent) to give the white solid 3-methyl-1-(2,2,2-trifluoromethyl)-1H-pyrazol-3-one (5a). Yield 60.2\%; m.p. $105-106{ }^{\circ} \mathrm{C} ;{ }^{1} \mathrm{H}-\mathrm{NMR}\left(\mathrm{CDCl}_{3}, 400 \mathrm{MHz}\right), \delta$ (ppm): 2.13 (s, 3H, $\left.\mathrm{CH}_{3}\right), 3.25$ (s, $2 \mathrm{H}$, $\left.\mathrm{CH}_{2}\right), 4.19-4.25\left(\mathrm{q}, 2 \mathrm{H}, \mathrm{J}=8.8 \mathrm{~Hz}, \mathrm{CH}_{2} \mathrm{CF}_{3}\right.$ ); ESI-MS [M + H] ${ }^{+}: 181.1$.

To a solution of ethyl acetoacetate $(0.03 \mathrm{~mol})$ in water $(12 \mathrm{~mL})$ and ethanol $(6 \mathrm{~mL})$ phenylhydrazine $(0.03 \mathrm{~mol})$ was added. After stirring for $2 \mathrm{~min}$ at room temperature, $1 \mathrm{~mL}$ of $36.5 \%$ concentrated hydrochloric acid was added and stirred at room temperature for $5 \mathrm{~min}$, then at $60{ }^{\circ} \mathrm{C}$ for $1.5 \mathrm{~h}$. The reaction mixture was treated with $10 \%$ sodium hydroxide solution to $\mathrm{pH} 7$, after stirring for $10 \mathrm{~min}$, the precipitate was filtered, washed with distilled water, and dried to give a light yellow solid as 3-methyl-1-phenyl-2-pyrazoline-5-one (5b). Yield 80.5\%; m.p. 126-127 ${ }^{\circ} \mathrm{C} ;{ }^{1} \mathrm{H}-\mathrm{NMR}\left(\mathrm{CDCl}_{3}, 400 \mathrm{MHz}\right)$, $\delta$ (ppm): $2.27\left(\mathrm{~s}, 3 \mathrm{H}, \mathrm{CH}_{3}\right), 3.43(\mathrm{~s}, 2 \mathrm{H}$, pyrazole- $4 \mathrm{H}), 7.18-7.23(\mathrm{~m}, 1 \mathrm{H}, \mathrm{ArH}), 7.37-7.43(\mathrm{~m}, 2 \mathrm{H}, \mathrm{ArH})$, $7.84-7.87(\mathrm{t}, 2 \mathrm{H}, J=8.8 \mathrm{~Hz}, \mathrm{ArH})$; ESI-MS $[\mathrm{M}+\mathrm{H}]^{+}: 175$. The compound $5 \mathbf{b}$ was a known compound and its experimental data in the literature [32,33] was shown as follows: m.p., $126-128{ }^{\circ} \mathrm{C},{ }^{1} \mathrm{H}-\mathrm{NMR}$ $\left(400 \mathrm{MHz}, \mathrm{CDCl}_{3}\right), \delta(\mathrm{ppm}): 2.13\left(\mathrm{~s}, 3 \mathrm{H}, \mathrm{CH}_{3}\right), 3.37(\mathrm{~s}, 2 \mathrm{H}$, pyrazole- $4 \mathrm{H}), 7.13(\mathrm{t}, J=7.6 \mathrm{~Hz}, 1 \mathrm{H}, \mathrm{ArH})$, $7.35(\mathrm{t}, J=8.0 \mathrm{~Hz}, 2 \mathrm{H}, \mathrm{ArH}), 7.81(\mathrm{~d}, J=7.6 \mathrm{~Hz}, 2 \mathrm{H}, \mathrm{ArH})$.

\subsubsection{Synthesis of Compounds $\mathbf{6} \mathbf{a}-\mathbf{6} \mathbf{i}$}

Compound $5 \mathrm{a}(1.5 \mathrm{mmol})$ and powdered potassium carbonate $(2.25 \mathrm{mmol})$ were added into dimethylsulfoxide ( $20 \mathrm{~mL}) .2$, 6-Dichlorobenzoxazole $(1.5 \mathrm{mmol})$ was added to the solution and the mixture was stirred at room temperature for $3 \mathrm{~h}$. The mixture was diluted with water $(20 \mathrm{~mL})$ and extracted with diethyl ether $(30 \mathrm{~mL} \times 2)$. The extract was washed with water and saturated brine, dried over anhydrous sodium sulfate, the filtrate was concentrated in vacuo. The residue was further purified by silica gel column chromatography (ethyl acetate/petroleum ether: $1 / 10$ as the eluent) to give compound $\mathbf{6 a}$ as a white solid. Compounds $\mathbf{6} \mathbf{b}-\mathbf{i}$ were synthesized using the same procedures.

6-Chloro-2-((1-(2,2,2-trifluoroethyl)-3-methyl-1H-pyrazol-5-yl)oxy)benzoxazole (6a), white solid, yield 69.6\%, m.p. 105-106 ${ }^{\circ} \mathrm{C}$; IR (KBr, $\left.v_{\max }, \mathrm{cm}^{-1}\right)$ : $2919\left(\mathrm{CH}_{3}\right), 1637$ (-C=N-), 1355 (C-F), 1260 (=C-O-C), 1164 (-C-O-C). ${ }^{1} \mathrm{H}-\mathrm{NMR}\left(\mathrm{CDCl}_{3}, 400 \mathrm{MHz}\right), \delta(\mathrm{ppm}): 2.24\left(\mathrm{~s}, 3 \mathrm{H}, \mathrm{CH}_{3}\right), 4.55-4.62(\mathrm{q}, 2 \mathrm{H}, J=8.24 \mathrm{~Hz}$, $\left.\mathrm{CH}_{2} \mathrm{CF}_{3}\right), 6.33(\mathrm{~s}, 1 \mathrm{H}$, pyrazole-4H), 7.25-7.27 (dd, $1 \mathrm{H}, J=8.5,1.8 \mathrm{~Hz}, \mathrm{ArH}), 7.42-7.43(\mathrm{~d}, 1 \mathrm{H}, J=1.8 \mathrm{~Hz}$, ArH), 7.43-7.45 (d, 1H, $J=8.6 \mathrm{~Hz}, \mathrm{ArH}) ;{ }^{13} \mathrm{C}-\mathrm{NMR}\left(\mathrm{CDCl}_{3}, 100 \mathrm{MHz}\right), \delta(\mathrm{ppm}): 13.57,47.60(\mathrm{q}$, $\left.{ }^{2} J_{\mathrm{C}, \mathrm{F}}=36 \mathrm{~Hz}\right), 92.38,110.04,118.87,121.65\left(\mathrm{q},{ }^{1} J_{\mathrm{C}, \mathrm{F}}=278 \mathrm{~Hz}\right), 124.69,128.97,137.74,145.39,147.39$, 148.73, 157.90; ESI-MS [M + H] $]^{+}$: 332.1. Anal. Calcd for $\mathrm{C}_{13} \mathrm{H}_{9} \mathrm{ClF}_{3} \mathrm{~N}_{3} \mathrm{O}_{2}: \mathrm{C}, 47.08 ; \mathrm{H}, 2.74 ; \mathrm{N}, 12.67$. Found: $\mathrm{C}, 47.11 ; \mathrm{H}, 2.72 ; \mathrm{N}, 12.68$. 
2-((1-(2,2,2-Trifluoroethyl)-3-methyl-1H-pyrazol-5-yl)oxy)benzoxazole (6b), white solid, yield 69.7\%, m.p. 83-84 ${ }^{\circ} \mathrm{C}$; IR (KBr, v max $\left.\mathrm{cm}^{-1}\right)$ : $2969\left(\mathrm{CH}_{3}\right), 1629$ (-C=N-), 1318 (C-F), 1257 (=C-O-C), 1175 (-C-O-C). ${ }^{1} \mathrm{H}-\mathrm{NMR}\left(\mathrm{CDCl}_{3}, 400 \mathrm{MHz}\right), \delta(\mathrm{ppm}): 2.31\left(\mathrm{~s}, 3 \mathrm{H}, \mathrm{CH}_{3}\right), 4.63-4.69\left(\mathrm{q}, 2 \mathrm{H}, \mathrm{J}=8.24 \mathrm{~Hz}, \mathrm{CH}_{2} \mathrm{CF}_{3}\right)$, 6.40 (s, 1H, pyrazole-4H), 7.29-7.36 (m, 2H, ArH), 7.45-7.48 (m, 1H, ArH), 7.59-7.61 (m, 1H, ArH); ${ }^{13} \mathrm{C}-\mathrm{NMR}\left(\mathrm{CDCl}_{3}, 100 \mathrm{MHz}\right), \delta(\mathrm{ppm}): 13.57,47.60\left(\mathrm{q},{ }^{2} J_{\mathrm{C}, \mathrm{F}}=36 \mathrm{~Hz}\right), 92.39,110.04,118.87,121.65$ (q, $\left.{ }^{1} J_{\mathrm{C}, \mathrm{F}}=278 \mathrm{~Hz}\right), 124.69,128.99,137.75,145.40,147.40,148.73,157.90 ; \mathrm{ESI}-\mathrm{MS}[\mathrm{M}+\mathrm{H}]^{+}$: 298.2. Anal. Calcd for $\mathrm{C}_{13} \mathrm{H}_{10} \mathrm{~F}_{3} \mathrm{~N}_{3} \mathrm{O}_{2}$ : C, 52.53; $\mathrm{H}, 3.39 ; \mathrm{N}, 14.14$; Found: $\mathrm{C}, 52.50 ; \mathrm{H}, 3.36 ; \mathrm{N}, 14.13$.

5-Chloro-2-((1-(2,2,2-trifluoroethyl)-3-methyl-1H-pyrazol-5-yl)oxy)benzoxazole (6c), white solid, yield 71.2\%, m.p. 85-86 ${ }^{\circ} \mathrm{C}$; IR (KBr, $\left.v_{\max }, \mathrm{cm}^{-1}\right)$ : $2924\left(\mathrm{CH}_{3}\right), 1623$ (-C=N-), 1307 (C-F), 1254 (=C-O-C), 1149 (-C-O-C). ${ }^{1} \mathrm{H}-\mathrm{NMR}\left(\mathrm{CDCl}_{3}, 400 \mathrm{MHz}\right), \delta$ (ppm): $2.33\left(\mathrm{~s}, 3 \mathrm{H}, \mathrm{CH}_{3}\right), 4.65-4.71(\mathrm{q}, 2 \mathrm{H}, \mathrm{J}=8.24 \mathrm{~Hz}$, $\left.\mathrm{CH}_{2} \mathrm{CF}_{3}\right), 6.42$ (s, 1H, pyrazole-4H), 7.30-7.33 (dd, 1H, J = 8.68, $\left.2.12 \mathrm{~Hz}, \mathrm{ArH}\right), 7.40-7.42(\mathrm{~d}, 1 \mathrm{H}$, $J=8.64 \mathrm{~Hz}, \mathrm{ArH}) ; 7.60-7.61(\mathrm{~d}, 1 \mathrm{H}, J=2.0 \mathrm{~Hz}, \mathrm{ArH}) ;{ }^{13} \mathrm{C}-\mathrm{NMR}\left(\mathrm{CDCl}_{3}, 100 \mathrm{MHz}\right), \delta(\mathrm{ppm}): 14.59,47.60$ $\left(q^{2} J_{C, F}=36 \mathrm{~Hz}\right), 93.44,111.05,119.51,122.88\left(q,{ }^{1} J_{C, F}=278 \mathrm{~Hz}\right), 124.72,130.61,141.14,146.35,146.92$, 149.75, 159.40; ESI-MS [M+ H] ${ }^{+}$: 332.1. Anal. Calcd for $\mathrm{C}_{13} \mathrm{H}_{9} \mathrm{ClF}_{3} \mathrm{~N}_{3} \mathrm{O}_{2}: \mathrm{C}, 47.08 ; \mathrm{H}, 2.74 ; \mathrm{N}, 12.67$; Found: C, 47.12; H, 2.72; N, 12.68 .

5-Chloro-2-((1-(2,2,2-trifluoroethyl)-3-methyl-1H-pyrazol-5-yl)oxy)pyrimidine (6d), yellow solid, yield 47.9\%, m.p. 56-57 ${ }^{\circ} \mathrm{C}$; IR (KBr, $\left.v_{\max }, \mathrm{cm}^{-1}\right)$ : $2968\left(\mathrm{CH}_{3}\right), 1626$ (-C=N-), 1307 (C-F), 1259 (=C-O-C). ${ }^{1} \mathrm{H}-\mathrm{NMR}\left(\mathrm{CDCl}_{3}, 400 \mathrm{MHz}\right), \delta(\mathrm{ppm}): 2.31$ (s, 3H, $\left.\mathrm{CH}_{3}\right), 4.60-4.67$ (q, 2H, J = 8.36 Hz, $\left.\mathrm{CH}_{2} \mathrm{CF}_{3}\right), 6.07$ (s, $1 \mathrm{H}$, pyrazole-4H), 8.59 (s, 2H, pyrimidine-4, 6H); ${ }^{13} \mathrm{C}-\mathrm{NMR}\left(\mathrm{CDCl}_{3}, 100 \mathrm{MHz}\right), \delta(\mathrm{ppm}): 13.57,47.42(\mathrm{q}$, $\left.{ }^{2} J_{\mathrm{C}, \mathrm{F}}=36 \mathrm{~Hz}\right), 93.23,121.77\left(\mathrm{q},{ }^{1} J_{\mathrm{C}, \mathrm{F}}=278 \mathrm{~Hz}\right), 126.24,146.36,148.47,157.16,159.74 ; \mathrm{ESI}-\mathrm{MS}[\mathrm{M}+\mathrm{H}]^{+}$: 293.1. Anal. Calcd for $\mathrm{C}_{10} \mathrm{H}_{8} \mathrm{ClF}_{3} \mathrm{~N}_{4} \mathrm{O}$ : C, 41.04; H, 2.76; N, 19.14; Found: C, 41.08; H, 2.75; N, 19.17 .

6-Chloro-2-((3-methyl-1-phenyl-1H-pyrazol-5-yl)oxy)benzoxazole (6e), white solid, yield 64.5\%, m.p. 78-79 ${ }^{\circ} \mathrm{C}$; IR $\left(\mathrm{KBr}, v_{\max }, \mathrm{cm}^{-1}\right): 2928\left(\mathrm{CH}_{3}\right), 1634$ (-C=N-), 1258 (=C-O-C), 1147 (-C-O-C). ${ }^{1} \mathrm{H}-\mathrm{NMR}$ $\left(\mathrm{CDCl}_{3}, 400 \mathrm{MHz}\right), \delta(\mathrm{ppm}): 2.40\left(\mathrm{~s}, 3 \mathrm{H}, \mathrm{CH}_{3}\right), 6.46(\mathrm{~s}, 1 \mathrm{H}$, pyrazole-4H), 7.31-7.37 (m, 2H, ArH), 7.45-7.52 (m, 4H, ArH), 7.63-7.65 (d, 2H, J = 7.6 Hz, ArH); ${ }^{13} \mathrm{C}-\mathrm{NMR}\left(\mathrm{CDCl}_{3}, 100 \mathrm{MHz}\right), \delta$ (ppm): 14.66, 94.53, 110.97, 119.80, 123.06, 125.57, 127.50, 129.22, 129.74, 137.64, 138.90, 145.08, 148.46, 149.11, 159.66; ESI-MS $[\mathrm{M}+\mathrm{H}]^{+}$: 326.1. Anal. Calcd for $\mathrm{C}_{17} \mathrm{H}_{12} \mathrm{ClN}_{3} \mathrm{O}_{2}: \mathrm{C}, 62.68 ; \mathrm{H}, 3.71 ; \mathrm{N}, 12.90 ;$ Found: $\mathrm{C}, 62.75 ; \mathrm{H}$, $3.69 ; \mathrm{N}, 12.92$.

2-((3-Methyl-1-phenyl-1H-pyrazol-5-yl)oxy)benzoxazole (6f), white solid, yield 58.9\%, m.p. $42-43{ }^{\circ} \mathrm{C}$; IR (KBr, v $\left.v_{\max }, \mathrm{cm}^{-1}\right): 2927\left(\mathrm{CH}_{3}\right), 1631$ (-C=N-), 1256 (=C-O-C), 1153 (-C-O-C). ${ }^{1} \mathrm{H}-\mathrm{NMR}\left(\mathrm{CDCl}_{3}\right.$, $400 \mathrm{MHz}), \delta$ (ppm): 2.40 (s, 3H, $\left.\mathrm{CH}_{3}\right), 6.47$ (s, 1H, pyrazole-4H), 7.29-7.36 (m, 3H, ArH), 7.44-7.49 (m, 3H, ArH), 7.59-7.61 (dd, 1H, J = 8.88, 1.64 Hz, ArH), 7.65-7.66 (d, 2H, J = 7.72 Hz, ArH); ${ }^{13} \mathrm{C}-\mathrm{NMR}$ $\left(\mathrm{CDCl}_{3}, 100 \mathrm{MHz}\right), \delta$ (ppm): 14.66, 94.54, 110.18, 119.26, 123.05, 124.21, 124.92, 127.40, 129.18, 137.63, 140.21, 145.34, 148.51, 149.10, 159.45; ESI-MS [M + H] ${ }^{+}$: 292.2. Anal. Calcd for $\mathrm{C}_{17} \mathrm{H}_{13} \mathrm{~N}_{3} \mathrm{O}_{2}$ : C, 70.09 ; H, 4.50; N, 14.42; Found: C, 70.05; H, 4.46; N, 14.42 .

5-Chloro-2-((3-methyl-1-phenyl-1H-pyrazol-5-yl)oxy)benzoxazole (6g), white solid, yield 49.3\%, m.p. 82-83 ${ }^{\circ} \mathrm{C}$; IR (KBr, v $\left.\max , \mathrm{cm}^{-1}\right): 2924\left(\mathrm{CH}_{3}\right), 1630$ (-C=N-), 1254 (=C-O-C), 1174 (-C-O-C). ${ }^{1} \mathrm{H}-\mathrm{NMR}$ $\left(\mathrm{CDCl}_{3}, 400 \mathrm{MHz}\right), \delta(\mathrm{ppm}): 2.40\left(\mathrm{~s}, 3 \mathrm{H}, \mathrm{CH}_{3}\right), 6.46(\mathrm{~s}, 1 \mathrm{H}$, pyrazole-4H), 7.26-7.28 (m, 1H, ArH), 7.33-7.38 (m, 2H, ArH), 7.45-7.49 (t, 2H, J = 7.34 Hz, ArH), 7.58 (d, 1H, J = 1.72 Hz, ArH), 7.63-7.65 $(\mathrm{d}, 2 \mathrm{H}, J=7.88 \mathrm{~Hz}, \mathrm{ArH}) ;{ }^{13} \mathrm{C}-\mathrm{NMR}\left(\mathrm{CDCl}_{3}, 100 \mathrm{MHz}\right), \delta$ (ppm): 14.68, 94.91, 121.47, 122.26, 122.90, 124.79, 126.59, 127.23, 129.15, 132.37, 137.80, 147.36, 148.48, 149.03, 168.84. ESI-MS [M + H] $]^{+} 326.1$. Anal. Calcd for $\mathrm{C}_{17} \mathrm{H}_{12} \mathrm{ClN}_{3} \mathrm{O}_{2}$ : C, 62.68; H, 3.71; N, 12.90; Found: C, 62.75; H, 3.69; N, 12.92 .

5-Chloro-2-((3-methyl-1-phenyl-1H-pyrazol-5-yl)oxy)pyrimidine (6h), yellow solid, yield 65.4\%, m.p. 49-50 ${ }^{\circ} \mathrm{C}$; IR (KBr, v $\left.v_{\max }, \mathrm{cm}^{-1}\right)$ : $2951\left(\mathrm{CH}_{3}\right), 1560$ (-C=N-), 1290 (=C-O-C), 758 (C-Cl). ${ }^{1} \mathrm{H}-\mathrm{NMR}$ $\left(\mathrm{CDCl}_{3}, 400 \mathrm{MHz}\right), \delta(\mathrm{ppm}): 2.39\left(\mathrm{~s}, 3 \mathrm{H}, \mathrm{CH}_{3}\right), 6.08(\mathrm{~s}, 1 \mathrm{H}$, pyrazole-4H), 7.24-7.28 (t, 1H, J = 7.48 Hz, $\mathrm{ArH}), 7.36-7.40(\mathrm{t}, 2 \mathrm{H}, \mathrm{J}=7.64 \mathrm{~Hz}, \mathrm{ArH}), 7.62-7.64(\mathrm{~d}, 2 \mathrm{H}, \mathrm{J}=7.68 \mathrm{~Hz}, \mathrm{ArH}), 8.48$ (s, 2H, pyrimidine-H); ${ }^{13} \mathrm{C}-\mathrm{NMR}\left(\mathrm{CDCl}_{3}, 100 \mathrm{MHz}\right), \delta$ (ppm): 14.65, 95.67, 122.68, 126.76, 126.96, 129.06, 138.07, 146.12, 149.02, 
158.09, 161.49; ESI-MS $[\mathrm{M}+\mathrm{H}]^{+}$: 287.1. Anal. Calcd for $\mathrm{C}_{14} \mathrm{H}_{11} \mathrm{ClN}_{4} \mathrm{O}: \mathrm{C}, 58.65 ; \mathrm{H}, 3.87 ; \mathrm{N}, 19.54$; Found: C, 58.72; H, 3.84; N, 19.57 .

2-((3-Methyl-1-phenyl-1H-pyrazol-5-yl)oxy)benzothiazole (6i), yellow solid, yield 65.7\%, m.p. 82-83 ${ }^{\circ} \mathrm{C}$; IR $\left(\mathrm{KBr}, v_{\max }, \mathrm{cm}^{-1}\right): 2925\left(\mathrm{CH}_{3}\right), 1558$ (-C=N-), 1217 (=C-O-C), 689(-C-S-C). ${ }^{1} \mathrm{H}-\mathrm{NMR}\left(\mathrm{CDCl}_{3}\right.$, $400 \mathrm{MHz}), \delta(\mathrm{ppm}): 2.40\left(\mathrm{~s}, 3 \mathrm{H}, \mathrm{CH}_{3}\right), 6.35(\mathrm{~s}, 1 \mathrm{H}$, pyrazole-4H), 7.30-7.36 (m, 2H, ArH), 7.43-7.47 $(\mathrm{m}, 3 \mathrm{H}, \mathrm{ArH}), 7.64-7.66(\mathrm{~d}, 2 \mathrm{H}, J=8.2 \mathrm{~Hz}, \mathrm{ArH}), 7.70-7.72(\mathrm{~d}, 1 \mathrm{H}, J=8.08 \mathrm{~Hz}, \mathrm{ArH}), 7.81-7.83(\mathrm{~d}, 1 \mathrm{H}$, $J=8.12 \mathrm{~Hz}, \mathrm{ArH}) ;{ }^{13} \mathrm{C}-\mathrm{NMR}\left(\mathrm{CDCl}_{3}, 100 \mathrm{MHz}\right), \delta$ (ppm): 14.67, 18.43, 94.90, 121.46, 122.27, 122.92, $124.79,126.59,127.24,129.15,132.37,137.79,147.36,148.48,149.04,168.85 ;$ ESI-MS [M + H] ${ }^{+}: 308.2$. Anal. Calcd for $\mathrm{C}_{17} \mathrm{H}_{13} \mathrm{~N}_{3} \mathrm{OS}$ : C, 66.43; H, 4.26; N, 13.67; Found: C, 66.40; H, 4.23; N, 13.67.

\subsubsection{Synthesis of Compounds 7a-7e}

Compound 6a $(0.5 \mathrm{mmol})$ was dissolved in $N, N$-Dimethylformamide (DMF, $6 \mathrm{~mL}$ ) and $\mathrm{N}$-bromosuccinimide (NBS, $0.6 \mathrm{mmol}$ ) was added in this solution, this mixture was stirred at room temperature overnight. Then, the mixture was poured into water $(10 \mathrm{~mL})$ and stored overnight, filtered, and the residue was the desired product $7 \mathbf{a}$. Compounds $7 \mathbf{b}-7 \mathbf{e}$ were synthesized using the same procedures.

2-((4-Bromo-1-(2,2,2-trifluoroethyl)-3-methyl-1H-pyrazol-5-yl)oxy)-6-chlorobenzoxazole (7a), white solid, yield 49.8\%, m.p. $131-132{ }^{\circ} \mathrm{C}$; IR (KBr, $\left.v_{\max }, \mathrm{cm}^{-1}\right)$ : $2982\left(\mathrm{CH}_{3}\right), 1625(-\mathrm{C}=\mathrm{N}-), 1375$ (C-F), 1253 (=C-O-C), 1170 (-C-O-C), 598 (C-Br). ${ }^{1} \mathrm{H}-\mathrm{NMR}\left(\mathrm{CDCl}_{3}, 400 \mathrm{MHz}\right), \delta$ (ppm): 2.64 (s, 3H, $\left.\mathrm{CH}_{3}\right), 4.93-4.99$ (q, $\left.2 \mathrm{H}, J=8.24 \mathrm{~Hz}, \mathrm{CH}_{2} \mathrm{CF}_{3}\right), 7.40-7.43(\mathrm{dd}, 1 \mathrm{H}, J=8.48,1.8 \mathrm{~Hz}, \mathrm{ArH}), 7.59(\mathrm{~d}, 1 \mathrm{H}, J=1.4 \mathrm{~Hz}$, $\mathrm{ArH}), 7.61-7.63(\mathrm{~d}, 1 \mathrm{H}, J=8.52 \mathrm{~Hz}, \mathrm{ArH}) ;{ }^{13} \mathrm{C}-\mathrm{NMR}\left(\mathrm{CDCl}_{3}, 100 \mathrm{MHz}\right), \delta(\mathrm{ppm}): 14.24,45.62(\mathrm{q}$, $\left.{ }^{2} J_{\mathrm{C}, \mathrm{F}}=35 \mathrm{~Hz}\right), 96.28,111.30,120.48,122.85\left(\mathrm{q},{ }^{1} J_{\mathrm{C}, \mathrm{F}}=280 \mathrm{~Hz}\right), 126.37,131.15,138.46,149.10,151.88$, 152.43, 163.48; ESI-MS $[\mathrm{M}+\mathrm{H}]^{+}: 410,412$. Anal. Calcd for $\mathrm{C}_{13} \mathrm{H}_{8} \mathrm{BrClF}_{3} \mathrm{~N}_{3} \mathrm{O}_{2}: \mathrm{C}, 38.03 ; \mathrm{H}, 1.96 ; \mathrm{N}$, 10.23; Found: C, 37.96; H, 1.95; N, 10.22 .

2-((4-Bromo-1-(2,2,2-trifluoroethyl)-3-methyl-1H-pyrazol-5-yl)oxy)benzoxazole (7b), white solid, yield 52.2\%, m.p. 107-108 ${ }^{\circ} \mathrm{C}$; IR (KBr, $\left.v_{\max }, \mathrm{cm}^{-1}\right)$ : $2925\left(\mathrm{CH}_{3}\right), 1622$ (-C=N-), 1319 (C-F), 1267 (=C-O-C), 1162 (-C-O-C), 745 (C-Br). ${ }^{1} \mathrm{H}-\mathrm{NMR}\left(\mathrm{CDCl}_{3}, 400 \mathrm{MHz}\right), \delta(\mathrm{ppm}): 2.28\left(\mathrm{~s}, 3 \mathrm{H}, \mathrm{CH}_{3}\right), 4.62-4.68(\mathrm{q}, 2 \mathrm{H}$, $\left.J=8.16 \mathrm{~Hz}, \mathrm{CH}_{2} \mathrm{CF}_{3}\right), 7.31-7.36(\mathrm{~m}, 2 \mathrm{H}, \mathrm{ArH}), 7.48-7.50(\mathrm{~m}, 1 \mathrm{H}, \mathrm{ArH}), 7.57-7.59(\mathrm{~m}, 1 \mathrm{H}, \mathrm{ArH})$; ${ }^{13} \mathrm{C}-\mathrm{NMR}\left(\mathrm{CDCl}_{3}, 100 \mathrm{MHz}\right), \delta(\mathrm{ppm}): 13.14,49.42\left(\mathrm{q},{ }^{2} J_{\mathrm{C}, \mathrm{F}}=36 \mathrm{~Hz}\right), 84.38,110.47,119.52,122.34(\mathrm{q}$, $\left.{ }^{1} J_{\mathrm{C}, \mathrm{F}}=278 \mathrm{~Hz}\right), 124.56,125.20,139.90,144.29,149.03,149.05,158.77$; ESI-MS [M + H] ${ }^{+}:$376.1, 378.1; Anal. Calcd for $\mathrm{C}_{13} \mathrm{H}_{9} \mathrm{BrF}_{3} \mathrm{~N}_{3} \mathrm{O}_{2}$ : C, 41.51; $\mathrm{H}, 2.41 ; \mathrm{N}, 11.17$; Found: $\mathrm{C}, 47.47 ; \mathrm{H}, 2.39 ; \mathrm{N}, 11.16$.

2-((4-Bromo-1-(2,2,2-trifluoroethyl)-3-methyl-1H-pyrazol-5-yl)oxy)-5-chlorobenzoxazole (7c), white solid, yield 83.2\%, m.p. $112-113{ }^{\circ} \mathrm{C}$; IR (KBr, $\left.v_{\max }, \mathrm{cm}^{-1}\right): 2955\left(\mathrm{CH}_{3}\right), 1627(-\mathrm{C}=\mathrm{N}-), 1349$ (C-F), 1253 (=C-O-C), 1172 (-C-O-C), 698 (C-Br). ${ }^{1} \mathrm{H}-\mathrm{NMR}\left(\mathrm{CDCl}_{3}, 400 \mathrm{MHz}\right), \delta(\mathrm{ppm}): 2.61$ (s, 3H, $\left.\mathrm{CH}_{3}\right), 4.91-4.97$ $\left(\mathrm{q}, 2 \mathrm{H}, J=8.36 \mathrm{~Hz}, \mathrm{CH}_{2} \mathrm{CF}_{3}\right), 7.38-7.40(\mathrm{dd}, 1 \mathrm{H}, J=8.52,1.92 \mathrm{~Hz}, \mathrm{ArH}), 7.56-7.57(\mathrm{~d}, 1 \mathrm{H}, J=1.84$ $\mathrm{Hz}, \mathrm{ArH}), 7.58-7.61(\mathrm{~d}, 1 \mathrm{H}, J=8.52 \mathrm{~Hz}, \mathrm{ArH}) ;{ }^{13} \mathrm{C}-\mathrm{NMR}\left(\mathrm{CDCl}_{3}, 100 \mathrm{MHz}\right), \delta(\mathrm{ppm}): 13.13,49.61$ (q, $\left.{ }^{2} J_{\mathrm{C}, \mathrm{F}}=36 \mathrm{~Hz}\right), 84.38,110.47,118.18,122.39\left(\mathrm{q},{ }^{1} J_{\mathrm{C}, \mathrm{F}}=278 \mathrm{~Hz}\right), 124.20,130.25,134.33,145.97,146.82$, 148.91, 168.23; ESI-MS [M + H] $]^{+}: 410.1$, 412.1. Anal. Calcd for $\mathrm{C}_{13} \mathrm{H}_{8} \mathrm{BrClF}_{3} \mathrm{~N}_{3} \mathrm{O}_{2}: \mathrm{C}, 38.03 ; \mathrm{H}, 1.96 ; \mathrm{N}$, 10.23. Found: $\mathrm{C}, 37.96 ; \mathrm{H}, 1.95 ; \mathrm{N}, 10.22$.

2-((4-Bromo-1-(2,2,2-trifluoroethyl)-3-methyl-1H-pyrazol-5-yl)oxy)-5-chloropyrimidine (7d), white solid, yield 86.4\%, m.p. $101-102{ }^{\circ} \mathrm{C}$; IR (KBr, $\left.v_{\max }, \mathrm{cm}^{-1}\right)$ : 2993( $\left.\mathrm{CH}_{3}\right), 1562(-\mathrm{C}=\mathrm{N}-), 1279(\mathrm{C}-\mathrm{F}), 1160$ (=C-O-C), $641(\mathrm{C}-\mathrm{Br}) .{ }^{1} \mathrm{H}-\mathrm{NMR}\left(\mathrm{CDCl}_{3}, 400 \mathrm{MHz}\right), \delta(\mathrm{ppm}): 2.27\left(\mathrm{~s}, 3 \mathrm{H}, \mathrm{CH}_{3}\right), 4.54-4.60(\mathrm{q}, 2 \mathrm{H}$, $\left.J=8.24 \mathrm{~Hz}, \mathrm{CH}_{2} \mathrm{CF}_{3}\right), 8.55\left(\mathrm{~s}, 2 \mathrm{H}\right.$, pyrimidine-4,6H); ${ }^{13} \mathrm{C}-\mathrm{NMR}\left(\mathrm{CDCl}_{3}, 100 \mathrm{MHz}\right), \delta(\mathrm{ppm}): 13.12$, $49.27\left(\mathrm{q},{ }^{2} J_{\mathrm{C}, \mathrm{F}}=36 \mathrm{~Hz}\right), 84.73,122.42\left(\mathrm{q},{ }^{1} J_{\mathrm{C}, \mathrm{F}}=278 \mathrm{~Hz}\right), 127.61,145.12,148.72,158.34,160.60$; ESI-MS $[\mathrm{M}+\mathrm{H}]^{+}: 371$, 373. Anal. Calcd for $\mathrm{C}_{10} \mathrm{H}_{7} \mathrm{BrClF}_{3} \mathrm{~N}_{4} \mathrm{O}: \mathrm{C}, 32.33 ; \mathrm{H}, 1.90 ; \mathrm{N}, 15.08$. Found: $\mathrm{C}, 32.26 ; \mathrm{H}$, $1.88 ; \mathrm{N}, 15.05$. 
5-(Benzyloxy)-4-bromo-3-methyl-1-phenyl-1H-pyrazole (7e), yellow liquid, yield 35.4\%; ${ }^{1} \mathrm{H}-\mathrm{NMR}\left(\mathrm{CDCl}_{3}\right.$, $400 \mathrm{MHz}), \delta(\mathrm{ppm}): 2.30\left(\mathrm{~s}, 3 \mathrm{H}, \mathrm{CH}_{3}\right), 5.18\left(\mathrm{~s}, 2 \mathrm{H}, \mathrm{CH}_{2}\right), 7.25-7.33(\mathrm{~m}, 6 \mathrm{H}, \mathrm{ArH}), 7.37-7.42(\mathrm{td}, 2 \mathrm{H}$, $J=7.4,1.88 \mathrm{~Hz}, \mathrm{ArH}), 7.53-7.54(\mathrm{~d}, 1 \mathrm{H}, J=0.88 \mathrm{~Hz}, \mathrm{ArH}), 7.55-7.56(\mathrm{~m}, 1 \mathrm{H}, \mathrm{ArH}) ;{ }^{13} \mathrm{C}-\mathrm{NMR}\left(\mathrm{CDCl}_{3}\right.$, $100 \mathrm{MHz}), \delta$ (ppm): 13.15, 75.96, 82.42, 122.62, 126.89, 128.49, 128.64, 128.76, 128.91, 134.99, 138.36, 147.49, 149.84; ESI-MS [M + H] $]^{+}: 343,345$. Anal. Calcd for $\mathrm{C}_{17} \mathrm{H}_{15} \mathrm{BrN}_{2} \mathrm{O}: \mathrm{C}, 59.49 ; \mathrm{H}, 4.41 ; \mathrm{N}, 8.16$. Found: $\mathrm{C}, 59.30 ; \mathrm{H}, 4.36 ; \mathrm{N}, 8.14$.

\subsection{Biological Evaluation}

\subsubsection{Inhibitory Effect of the Target Compounds on the Growth of Weed Roots and Shoots}

Solutions of $1 \mathrm{~g} / \mathrm{L}$ and $10 \mathrm{~g} / \mathrm{L}$ of the tested compounds in DMF were prepared. Agar powder $(5 \mathrm{~g})$ was put into boiling distilled water $(1 \mathrm{~L})$ until it dissolved, and then cooled down to $40-50{ }^{\circ} \mathrm{C}$. The solution $(0.2 \mathrm{~mL})$ containing testing compound and melting agar $(19.8 \mathrm{~mL})$ was mixed, and this mixture was added to a $120 \mathrm{~mL}$ cup with $4.5 \mathrm{~cm}$ diameter. The agar plate without test compound was used as an untreated control. The 15 seeds of B. campestris, A. retroflexus, P. oleracea, P. alopecuroides, E. crus-galli, and D. sanguinalis were put on the surface of the agar plate. These cups were covered with glass lids, and the cultivation conditions were kept at $25 \pm 1{ }^{\circ} \mathrm{C}, 50 \%-55 \%$ relative humidity, and $12 \mathrm{~h}$ in the light and $12 \mathrm{~h}$ in the dark alternating for seven days. The experiments were conducted in three replicates. Seven days later, the roots lengths of B. campestris, A. retroflexus, P. oleracea, and $D$. sanguinalis and the shoots lengths of P. alopecuroides, and E. crus-galli were measured. The growth inhibitory rate related to untreated control was determined.

\subsubsection{Treatment in Greenhouse Conditions}

Twenty seeds of test plants were planted $(0.6 \mathrm{~cm}$ depth) in plastic boxes $(9 \mathrm{~cm}$ diameter) containing sandy soil. The plastic boxes were placed at $22-25^{\circ} \mathrm{C}$ in a greenhouse. The experiments were conducted in three replicates. The seedlings (one leaf and one stem) of the dicotyledonous weed and the seedlings (two leaf and one stem) of the monocotyledonous weed were sprayed with the test compounds at the concentration of $750 \mathrm{~g}$ a. i. ha ${ }^{-1}$. The emulsions of tested compounds were prepared by dissolving them in DMF with the addition proper water contained $0.1 \%$ Triton X-100. The fresh weights were determined 15 days later, and the percentage inhibition relative to the water-sprayed controls was calculated.

\subsubsection{Inhibitory Effect of the Compound $\mathbf{6} \mathbf{d}$ on Chlorophyll of Weeds}

To evaluate the bleaching activity of compound $\mathbf{6 d}$, the changes in chlorophyll contents of treated seedlings were evaluated by Arnon's method [34] as modified by Lichtenthaler [35]. Bleached seedlings of B. campestris, P. alopecuroides, E. crus-galli, and D. sanguinalis with different concentrations were obtained by the method described above. Chlorophyll a and b were extracted from $50 \mathrm{mg}$ of leaf tissue per treatment in $8 \mathrm{~mL}$ of $80 \%$ acetone in water for $24 \mathrm{~h}$. The absorbance was measured at $663 \mathrm{~nm}$ and $646 \mathrm{~nm}$, respectively. The contents of chlorophyll $\mathrm{a}$ and $\mathrm{b}$ in leaf tissue were finally calculated by the following formula: for chlorophyll a, $\mathrm{Ca}=12.21 A_{663}-2.81 A_{646}$; for chlorophyll $\mathrm{b}$, $C_{\mathrm{b}}=20.13 A_{646}-5.03 A_{663}$. The concentration causing $50 \%$ inhibition $\left(\mathrm{IC}_{50}\right)$ of in vitro activity for the selected compounds were obtained by analyzing inhibition curves of the activity values (\%) versus the logarithm of inhibitory concentration. Diflufenican was selected as the positive control. At least five doses in the inhibitory range were considered, and three replicates were performed under the same conditions. 


\section{Conclusions}

In conclusion, the design, synthesis, and structure-activity relationships of a series of pyrazoyl derivatives have been described. Some compounds displayed an efficient bleaching effect and herbicidal activities against monocotyledonous and dicotyledonous weeds. The herbicidal tests showed that when the 5-position of the pyrazole ring was substituted by heterocycle, the corresponding compounds presented herbicidal activities, especially compound $\mathbf{6} \mathbf{d}$ possessed good herbicidal activity against $D$. sanguinalis at the dosage of $750 \mathrm{~g}$ a. i. ha ${ }^{-1}$.

Supplementary Materials: The following ${ }^{1} \mathrm{H}-\mathrm{NMR}$ and ${ }^{13} \mathrm{C}-\mathrm{NMR}$ spectra are available online at http://www.mdpi.com/1420-3049/21/1/39/s1.

Acknowledgments: This work was financially supported by the Special Fund for Agro-scientific Research in the Public Interest (Program No.201303031), the National Natural Science Foundation of China (Program No. 31201554), and the Key Project in the National Science and Technology Pillar Program of China (Program No. 2012BAD27B00).

Author Contributions: J.K., X.L.Y., C.S.C. and J.H.L. performed research and analyzed the data; and H.J.M. performed research, analyzed the data and wrote the paper. All authors read and approved the final manuscript.

Conflicts of Interest: The authors declare no conflict of interest.

\section{References}

1. Ghorab, M.M.; Ragab, F.A.; Alqasoumi, S.I.; Alafeefy, A.M.; Aboulmagd, S.A. Synthesis of some new pyrazolo[3,4- $d]$ pyrimidine derivatives of expected anticancer and radio-protective activity. Eur. J. Med. Chem. 2010, 45, 171-178. [CrossRef] [PubMed]

2. Rostom, S.A.; Shalaby, M.A.; El-Demellawy, M.A. Polysubstituted pyrazoles, part 5. Synthesis of new 1-(4-chlorophenyl)-4-hydroxy-1H-pyrazole-3-carboxylic acid hydrazide analogs and some derived ring systems. A novel class of potential antitumor and anti-HCV agents. Eur. J. Med. Chem. 2003, 38, 959-974. [CrossRef] [PubMed]

3. Amnerkar, N.D.; Bhusari, K.P. Synthesis, anticonvulsant activity and 3D-QSAR study of some prop-2-eneamido and 1-acetyl-pyrazolin derivatives of aminobenzothiazole. Eur. J. Med. Chem. 2010, 45, 149-159. [CrossRef] [PubMed]

4. Yeu, J.P.; Yeh, J.T.; Chen, T.Y.; Uang, B.J. An expedient synthesis of 1-[3-(dimethylamino)propyl]5-methyl-3-phenyl-1H-indazole(FS-32)-An antidepressant. Synthesis 2001, 12, 1775-1777. [CrossRef]

5. Sharma, P.K.; Kumar, S.; Kumar, P.; Kaushik, P.; Kaushik, D.; Dhingra, Y.; Aneja, K.R. Synthesis and biological evaluation of some pyrazolylpyrazolines as anti-inflammatory-antimicrobial agents. Eur. J. Med. Chem. 2010, 45, 2650-2655. [CrossRef] [PubMed]

6. Kalaria, P.N.; Satasia, S.P.; Raval, D.K. Synthesis, identification and in vitro biological evaluation of some novel 5-imidazopyrazole incorporated pyrazoline and isoxazoline derivatives. New J. Chem. 2014, 38, 2902-2910. [CrossRef]

7. Shiga, Y.; Okada, I.; Fukuchi, T. Synthesis and acaricidal activity of N-(1,3,4-thiadiazol-2-yl)pyrazole5-carboxamides and N-(1,3,4-thiadiazol-2-yl) thiazole-5-carboxamides. J. Pestic. Sci. 2003, 28, 310-312. [CrossRef]

8. Shiga, Y.; Okada, I.; Ikeda, Y.; Takizawa, E.; Fukuchi, T. Insecticidal activity of $\mathrm{N}$-acy-N-(4-aryloxybenzyl)pyrazole-5-carboxamides. J. Pestic. Sci. 2003, 28, 313-314. [CrossRef]

9. Ohno, R.; Watanabe, A.; Matsukawa, T.; Ueda, T.; Sakurai, H.; Hori, M.; Hirai, K. Synthesis and herbicidal activity of new pyrazole-4-carboxamide derivatives. J. Pestic. Sci. 2004, 29, 15-26. [CrossRef]

10. Vicentini, C.B.; Mares, D.; Tartari, A.; Manfrini, M.; Forlani, G. Synthesis of pyrazole derivatives and their evaluation as photosynthetic electron transport inhibitors. J. Agric. Food Chem. 2004, 52, 1898-1906. [CrossRef] [PubMed]

11. Vicentini, C.B.; Romagnoli, C.; Andreotti, E.; Mares, D. Synthetic pyrazole derivatives as growth inhibitors of some phytopathogenic fungi. J. Agric. Food Chem. 2007, 55, 10331-10338. [CrossRef] [PubMed]

12. Li, H.B.; Zhu, Y.Q.; Song, X.W.; Hu, F.Z.; Liu, B.; Li, Y.H.; Niu, Z.X.; Liu, P.; Wang, Z.H.; Song, H.B.; et al. Novel protoporphyrinogen oxidase inhibitors: $3 H$-pyrazolo[3,4-d]-[1,2,3]triazin-4-one derivatives. J. Agric. Food Chem. 2008, 56, 9535-9542. [CrossRef] [PubMed] 
13. Ouyang, G.P.; Cai, X.J.; Chen, Z.; Song, B.A.; Bhadury, P.S.; Yang, S.; Jin, L.H.; Xue, W.; Hu, D.Y.; Zeng, S. Synthesis and antiviral activities of pyrazole derivatives containing an oxime moiety. J. Agric. Food Chem. 2008, 56, 10160-10167. [CrossRef] [PubMed]

14. Ouyang, G.P.; Chen, Z.; Cai, X.J.; Song, B.A.; Bhadury, P.S.; Yang, S.; Jin, L.H.; Xue, W.; Hu, D.Y.; Zeng, S. Synthesis and antiviral activity of novel pyrazole derivatives containing oxime esters group. Bioorg. Med. Chem. 2008, 16, 9699-9707. [CrossRef] [PubMed]

15. Hirai, K.; Uchida, A.; Ohno, R. Herbicide Classes in Development; Boger, P., Hirai, K., Wakabyashi, K., Eds.; Springer-Verlag: Heidelberg, Germany, 2002; pp. 179-289.

16. Wang, H.X.; Wu, L.L.; Wang, Y.M.; Zhou, Z.H. Organocatalyzed asymmetric tandem Michaelcyclization reaction of 4-benzylidene-3-methylpyrazol-5-ones and malononitrile: Stereocontrolled construction of pyrano[2,3-c]pyrazole scaffold. RSC Adv. 2015, 5, 42836-42842. [CrossRef]

17. Moon, J.K.; Kim, J.H.; Shibamoto, T. Photodegradation Pathways and mechanisms of the herbicide metamifop in a water/acetonitrile solution. J. Agric. Food Chem. 2010, 58, 12357-12365. [CrossRef] [PubMed]

18. Kalaria, P.N.; Satasia, S.P.; Raval, D. K. L-Proline promoted green and regioselective synthesis of a novel pyrazole based trifluoromethylated fused thiazolopyran scaffold and its biological evaluation. RSC Adv. 2014, 4, 32353-32362. [CrossRef]

19. Roe, R.M.; Burton, J.D.; Kuhr, R.J. Herbicide Activity: Toxicology, Biochemistry and Molecular Biology; Sandmann, G., Böger, P., Eds.; IOS Press: Amsterdam, The Netherlands, 1997; pp. 111-141.

20. Sandmann, G. Bleaching activities of substituted pyrimidines and structure-activity comparison to related heterocyclic derivatives. Pestic. Biochem. Physiol. 2001, 70, 86-91. [CrossRef]

21. Ogawa, H.; Yamada, I.; Arai, K.; Hirase, K.; Moriyasu, K.; Schneider, C.; Sandmann, G.; Boger, P.; Wakabayashi, K. Mode of Bleaching Phytotoxicity of Herbicidal Diphenylpyrrolidinones. Pest Manag. Sci. 2001, 57, 33-40. [CrossRef]

22. Ohki, S.; Miller-Sulger, R.; Wakabayashi, K.; Pfleiderer, W.; Böger, P. Phytoene desaturase inhibition by O-(2-phenoxy)ethyl-N-aralkylcarbamates. J. Agric. Food Chem. 2003, 51, 3049-3055. [CrossRef] [PubMed]

23. Laber, B.; Usunow, G.; Wiecko, E.; Franke, W.; Frank, H.; Köhn, A. Inhibition of narcissus pseudonarcissus phytoene desaturase by herbicidal 3-trifluoromethyl-1,1-biphenyl derivatives. Pestic. Biochem. Physiol. 1999, 63, 173-184. [CrossRef]

24. Krämer, W.; Schirmer, U.; Jeschke, P.; Witschel, M. Herbicides with bleaching properties. In Modern Crop Protection Compounds; John Wiley \& Sons: Malden, MA, USA, 2012; pp. 197-276.

25. Xu, H.; Zou, X.M.; Zhu, Y.Q. Synthesis and herbicidal activity of novel $\alpha, \alpha, \alpha$-trifluoro-m-tolyl pyridazinone derivatives. Pest. Manag. Sci. 2006, 62, 522-530. [CrossRef] [PubMed]

26. Xu, H.; Hu, X.H.; Zou, X.M.; Zhu, Y.Q.; Liu, B.; Hu, F.Z.; Yang, H.Z. Synthesis and herbicidal activity of 5-heterocycloxy-3-substituted-1-(3-trifluoromethyl)phenyl-1H-pyrazole. Chem. Res. Chin. Univ. 2012, 28, 824-827.

27. Kohn, A.; Franke, H.; Franke, W.; Bohner, J.; Rees, R. Substituted Biphenyl Derivatives and Their Use as Herbicides. EP Patent WO1996006089 A1, 29 February 1996.

28. Welch, J.T. Tetrahedron report number 221: Advances in the preparation of biologically active organofluorine compounds. Tetrahedron 1987, 43, 3123-3197. [CrossRef]

29. Ma, H.J.; Li, Y.H.; Zhao, Q.F.; Zhang, T.; Xie, R.L. Synthesis and herbicidal activity of novel N-(2,2,2)-trifluoroethylpyrazole derivatives. J. Agric. Food Chem. 2010, 58, 4356-4360. [CrossRef] [PubMed]

30. Ma, H.J.; Zhang, J.H.; Xia, X.D.; Xu, M.H.; Nin, J. Design, synthesis and herbicidal activities of novel 4-(1H-pyrazol-1-yl)-6-(alkynyloxy)-pyrimidine derivatives as potential pigment biosynthesis inhibitors. Pest Manag. Sci. 2014, 70, 946-952. [CrossRef] [PubMed]

31. Ma, H.J.; Zhang, J.H.; Xia, X.D.; Kang, J.; Li, J.H. Design, synthesis and herbicidal evaluation of novel 4-(1H-pyrazol-1-yl)pyrimidine derivatives. Pest Manag. Sci. 2015, 71, 1189-1196. [CrossRef] [PubMed]

32. Masoud, M.S.; Mostafa, M.A.; Ahmed, R.H.; Moneim, N.H.A. Structures and chemical equilibria of some $\mathrm{N}$-Heterocycles, containing amide linkages. Molecules 2003, 8, 430-438. [CrossRef]

33. Shimomura, O.; Lee, B.S.; Meth, S.; Suzuki, H.; Mahajan, S.; Nomura, R.; Janda, K.D. Synthesis and application of polytetrahydrofuran-grafted polystyrene (PS-PTHF) resin supports for organic synthesis. Tetrahedron 2005, 61, 12160-12167. [CrossRef]

34. Arnon, D.I. Copper enzymes in isolated chloroplasts polyphenoloxidase in Beta vulgaris. Plant Physiol. 1949, 24, 1-15. [CrossRef] [PubMed] 
35. Lichtenthaler, H.K.; Wellburn, A.R. Determinations of total carotenoids and chlorophylls a and b of leaf extracts in different solvents. Biochem. Soc. Trans. 1983, 11, 591-592. [CrossRef]

Sample Availability: Samples of the compounds 6a-6i are available from the authors.

(C) 2015 by the authors; licensee MDPI, Basel, Switzerland. This article is an open access article distributed under the terms and conditions of the Creative Commons by Attribution (CC-BY) license (http://creativecommons.org/licenses/by/4.0/). 